\title{
NEAT1/miR-124/STAT3 feedback loop promotes breast cancer progression
}

\author{
YAMEI PANG ${ }^{1 *}$, JIE WU $^{2 *}$, XIANG LI $^{2 *}$, CUICUI WANG ${ }^{3}$, \\ MENG WANG ${ }^{2}$, JIAN LIU ${ }^{2}$ and GANGHUA YANG ${ }^{4}$ \\ Departments of ${ }^{1}$ Respiratory and Critical Care Medicine and ${ }^{2}$ Thoracic Surgery, \\ The First Affiliated Hospital of Xi'an Jiaotong University, Xi'an, Shaanxi 710061; \\ ${ }^{3}$ Department of Hematology, Zoucheng People's Hospital, Zoucheng, \\ Shandong 273500; ${ }^{4}$ Department of Geriatric Surgery, \\ The First Affiliated Hospital of Xi'an Jiaotong University, \\ Xi'an, Shaanxi 710061, P.R. China
}

Received January 10, 2019; Accepted June 25, 2019

DOI: $10.3892 /$ ijo.2019.4841

\begin{abstract}
The long non-coding RNA nuclear enriched abundant transcript 1 (NEAT1) has important roles in the regulation of multiple cell functions, such as proliferation, apoptosis and migration. However, the mechanism by which NEAT1 regulates breast cancer progression is not well elucidated. In the present study, NEAT1 and microRNA-124 (miR-124) levels were detected by reverse transcription-quantitative PCR in breast cancer tissues and cell lines. STAT3 protein levels were detected by western blot analysis. Cell proliferation and cell cycle distribution were determined using MTT and colony formation assays, and flow cytometry, respectively. The results demonstrated that NEAT1 and STAT3 expression levels were increased in breast cancer tissues compared with normal breast tissues, whereas miR-124 expression was significantly decreased. Functional analyses revealed that NEAT1 promoted cell proliferation and cell cycle progression in breast cancer cells. Additionally, NEAT1 and STAT3 expression levels were negatively
\end{abstract}

Correspondence to: Dr Ganghua Yang, Department of Geriatric Surgery, The First Affiliated Hospital of Xi'an Jiaotong University, 277 Yanta West Road, Xian, Shaanxi 710061, P.R. China

E-mail: dr_yanggh@163.com

${ }^{*}$ Contributed equally

Abbreviations: NEAT1, nuclear enriched abundant transcript 1; lncRNA, long non-coding RNA; miR, microRNA; ceRNA, competing endogenous lncRNA; RT-qPCR, reverse transcription-quantitative PCR; NAT, normal adjacent breast tissue; NC, negative control; siRNA, small interfering RNA

Key words: nuclear enriched abundant transcript 1, microRNA-124, STAT3, breast cancer correlated with miR-124 levels in breast cancer tissues. A direct interaction between miR-124, and NEAT1 and STAT3, was predicted by bioinformatics analysis and confirmed using a luciferase activity assay. NEAT1 overexpression markedly increased STAT3 protein expression levels, and this effect was reversed by miR-124 overexpression in breast cancer cells. Furthermore, miR-124 overexpression partially attenuated the effects of NEAT1 on breast cancer cell proliferation and cell cycle progression. The inhibitory effects of miR-124 overexpression on the proliferation rate and cell cycle progression were abolished by STAT3 overexpression. In turn, STAT3 silencing inhibited NEAT1 transcription in breast cancer cells. In summary, the present findings revealed that NEAT1 and STAT3 formed a feedback loop via sponging miR-124 to promote breast cancer progression.

\section{Introduction}

Breast cancer is one of the most common malignant cancers and the leading cause of cancer-related mortality in women globally (1). Breast cancer is a heterogeneous disease generally divided into four major molecular subtypes: Luminal A and luminal B [which are mostly positive for estrogen receptor (ER) and progesterone receptor (PR) expression], triple negative breast cancer (TNBC)/basal-like and erb-b2 receptor tyrosine kinase 2 (HER2)-positive (2). Despite major developments in the diagnostic and therapeutic strategies, the prognosis of women with advanced breast cancer requires improvement (3). As a result, there is an urgent need to further elucidate the underlying molecular mechanisms of breast cancer progression.

Long non-coding RNAs (lncRNAs) are RNA molecules $>200$ nucleotides in length that do not have significant protein-coding potential (4). Recent studies have revealed that lncRNAs are involved in regulating multiple biological processes, including development, differentiation and carcinogenesis $(5,6)$. Various lncRNAs have been reported to participate in the progression of breast cancer. For example, $\mathrm{X}$ inactive specific 
transcript suppresses breast cancer cell growth, migration, and invasion via the microRNA (miR)-155/caudal type homeobox 1 axis (7). HOX transcript antisense RNA increases ligand-independent ER activities and contributes to tamoxifen resistance in breast cancer (8). H19 imprinted maternally expressed transcript, let-7 and RNA-binding protein LIN28 form a double-negative feedback loop and have an important role in the maintenance of breast cancer stem cells (9). Nuclear enriched abundant transcript 1 (NEAT1), also known as $\mathrm{MEN \varepsilon / \beta}$, is a novel lncRNA localized to nuclear paraspeckles. It is critical for the maintenance of paraspeckles and associated with the development of several types of cancer (10). A previous study demonstrated that NEAT1 promotes breast cancer progression by targeting the miR-448/zinc finger E-box binding homeobox 1 axis (11); however, the precise mechanisms by which NEAT1 promotes breast cancer progression remain largely unknown.

miRNAs are small, non-coding RNA molecules that regulate many cellular activities by binding the 3 '-untranslated region (3'-UTR) of corresponding mRNAs (12). Increasing evidence has indicated that dysregulation of miRNAs has an important role in cancer development $(13,14)$. Previous studies have demonstrated that miR-124 exerts a tumor suppressive role in various malignancies, including gastric cancer (15), hepatocellular carcinoma (16), bladder cancer (17) and non-small cell lung cancer (18). Notably, miR-124 overexpression inhibits cell growth, migration, invasion and chemoresistance in breast cancer (19-21). Furthermore, miR-124 overexpression enhances the sensitivity of HER2-positive breast cancer cells to irradiation by directly targeting STAT3 (22). However, the association between NEAT1, miR-124 and STAT3 in breast cancer is not fully understood.

The current study aimed to investigate the interaction between NEAT1, miR-124 and STAT3 in breast cancer. It was demonstrated that NEAT1 acts as a competing endogenous IncRNA (ceRNA) to positively regulate STAT3 by sponging miR-124. Furthermore, NEAT1 and STAT3 functioned coordinately to promote breast cancer progression by forming a positive feedback loop.

\section{Materials and methods}

Cell culture. To gain comprehensive data of gene expression in breast cancer cell lines, four cell lines were used in the present study to cover three common breast cancer subtypes: the ER/PR ${ }^{+}$cell lines MCF-7 and T47D; the HER2 ${ }^{+}$cell line SKBR3; and the TNBC cell line MDA-MB-231 (23). The breast cancer cell lines and the normal breast cell line MCF-10A were obtained from the American Type Culture Collection. The breast cancer cells were cultured in DMEM (Hyclone; GE Healthcare Life Sciences) supplemented with $10 \%$ FBS (Gibco; Thermo Fisher Scientific, Inc.) in a $5 \% \mathrm{CO}_{2}$ environment at $37^{\circ} \mathrm{C}$. The MCF-10A cell line was cultured in DMEM/F12 (1:1; Hyclone; GE Healthcare Life Sciences) supplemented with 5\% FBS, $10 \mu \mathrm{g} / \mathrm{ml}$ insulin, $20 \mathrm{ng} / \mathrm{ml}$ epidermal growth factor (EGF), $100 \mathrm{ng} / \mathrm{ml}$ cholera toxin and $0.5 \mathrm{mg} / \mathrm{ml}$ hydrocortisone at $37^{\circ} \mathrm{C}$ with $5 \% \mathrm{CO}_{2} . \mathrm{MCF}-7$ and MDA-MB-231 cells were used as representative for $\mathrm{ER}^{+}$and ER breast cancer, respectively, for subsequent functional assays.
Cell transfection. miR-124 mimics, miR-124 inhibitors, NEAT1 small interfering RNA (siRNA), STAT3 siRNA and the corresponding negative controls were synthesized by GenePharma Co., Ltd (the sequences of the oligonucleotides are provided in Table I). The full-length sequences of NEAT1 or STAT3 were amplified by PCR and subcloned into the pcDNA3.1 vector (Invitrogen; Thermo Fisher Scientific, Inc.) to generate the pcDNA-NEAT1 (NEAT1) or pcDNA-STAT3 (STAT3) overexpression plasmids, respectively. NEAT1, STAT3 and miR-124 levels were overexpressed by transfection with the NEAT1-overexpressing vector, STAT3-overexpressing vector and miR-124 mimics (50 $\mathrm{nM})$. NEAT1, STAT3 and miR-124 levels were depleted by transfection with NEAT1 siRNA, STAT3 siRNA and miR-124 inhibitors (100 nM). All transfections were performed using Lipofectamine ${ }^{\circledR} 2000$ reagent (Invitrogen; Thermo Fisher Scientific, Inc.) according to the manufacturer's instructions. Total RNA and protein were extracted at 48 or $72 \mathrm{~h}$ post-transfection, respectively.

Clinical samples. All clinical samples (31 pairs of matched breast cancer and normal breast tissue samples; age, 29-65) were obtained from the First Affiliated Hospital of Xi'an Jiaotong University (Xi'an, China) between November 2016 and December 2017. The study was approved by the Ethics Committee of Xi'an Jiaotong University First Affiliated Hospital and each patient provided written informed consent. The specimens were resected and frozen in liquid nitrogen immediately after surgery. None of the patients had received any preoperative local or systemic treatment.

Reverse transcription-quantitative PCR (RT-qPCR). Total RNA was extracted from surgical specimens and cultured cells using RNAiso Plus (Takara Biotechnology Co., Ltd.) according to the manufacture's protocol. Reverse transcription was performed using PrimeScript ${ }^{\mathrm{TM}}$ RT Reagent kit (Takara Biotechnology Co., Ltd.) as previously described (24). PCR was conducted using SYBR ${ }^{\circledR}$ Premix Ex Taq ${ }^{\mathrm{TM}}$ II (Tli RNaseH Plus2X; Takara Biotechnology Co., Ltd.) on a CFX96TM Real-Time PCR Detection System (Bio-Rad Laboratories, Inc.). The thermocycling conditions were as follows: $30 \mathrm{sec}$ at $95^{\circ} \mathrm{C}$, followed by 40 cycles of $5 \mathrm{sec}$ at $95^{\circ} \mathrm{C}$ and $30 \mathrm{sec}$ at $60^{\circ} \mathrm{C}$. $\beta$-actin was used as internal control for NEAT1 and STAT3. U6 was used as internal control for miR-124. The relative expression levels were calculated using the $2^{-\Delta \Delta \mathrm{Cq}}$ method (25). Five normal breast tissue samples derived from breast cancer patients were used as the normal control. The PCR primers used in this study are provided in Table II.

Western blot analysis. Total protein was extracted from cells by using a Total Protein Extraction kit (Nanjing KeyGen Biotech Co., Ltd.), according to the manufacturer's instructions. Measurement of protein concentration was conducted with a protein bicinchoninic acid assay kit (Thermo Fisher Scientific, Inc.). The total protein extracts $(20 \mu \mathrm{g})$ were separated by $10 \%$ SDS-PAGE and transferred onto nitrocellulose membranes (Bio-Rad Laboratories, Inc.). Non-specific binding sites were blocked by incubation in 5\% non-fat milk for $2 \mathrm{~h}$ at room temperature. Subsequently, the membranes were incubated with anti-STAT3 antibody (1:1,000; cat. no. ab68153; Abcam) 
Table I. Sequences of oligonucleotides used in this study.

\begin{tabular}{ll}
\hline Oligo & \\
\hline miR-124 mimics (sense) & Sequence (5'-3') \\
miR-124 mimics (antisense) & UAAGGCACGCGGUGAAUGCC \\
miR-124 inhibitor & CAUUCACCGCGUGCCUUAUU \\
NEAT1 siRNA (sense) & GGCAUUCACCGCGUGCCUUA \\
NEAT1 siRNA (antisense) & GUGAGAAGUUGCUUAGAAACUUUCC \\
STAT3 siRNA (sense) & GGAAAGUUUCUAAGCAACUUCUCAC \\
STAT3 siRNA (antisense) & GAAGGAGGCGUCACUUUCA \\
\end{tabular}

miR-124, microRNA-124; siRNA, small interfering RNA; NEAT, nuclear enriched abundant transcript 1.

Table II. Primers used for reverse transcription-quantitative PCR analysis.

\begin{tabular}{ll}
\hline Primer & \multicolumn{1}{c}{ Sequence (5'-3') } \\
\hline NEAT1, F & TGGCTAGCTCAGGGCTTCAG \\
NEAT1, R & TCTCCTTGCCAAGCTTCCTTC \\
STAT3, F & ATCACGCCTTCTACAGACTGC \\
STAT3, R & CATCCTGGAGATTCTCTACCACT \\
ACTB, F & CCTTCTACAATGAGCTGCGT \\
ACTB, R & CCTGGATAGCAACGTACATG \\
miR-124, F & GCGGCCGTGTTCACAGCGGACC \\
miR-124, R & GTGCAGGGTCCGAGGT \\
U6, F & GCTTCGGCAGCACATATACTAAAAT \\
U6, R & CGCTTCACGAATTTGCGTGTCAT \\
\hline
\end{tabular}

NEAT, nuclear enriched abundant transcript $1 ; \mathrm{F}$, forward; R, reverse; ACTB, $\beta$-actin; miR-124, microRNA-124.

at $4^{\circ} \mathrm{C}$ overnight. The membranes were then incubated with horseradish peroxidase-conjugated secondary antibody (1:5,000; cat. no. sc2004; Santa Cruz Biotechnology, Inc.). An anti- $\beta$-actin antibody (1:5,000; cat. no. A5441; Sigma-Aldrich; Merck KGaA) was used as the loading control. Protein signals were visualized using an enhanced chemiluminescence kit (EMD Millipore).

Luciferase activity assay. The putative binding sites for miR-124 were predicted using Targetscan (targetscan.org/mamm_31/), StarBase (starbase.sysu.edu.cn/) and miRcode (mircode. org/). The fragments containing the wild-type or mutant miR-124-binding sites of NEAT1 and STAT3 3'-UTRs were cloned into the pGL3-control vector (Promega Corporation) at the NheI and XhoI restriction sites to construct the luciferase reporter vectors. The putative STAT3-binding sites in the NEAT1 promoter were identified by using the University of California Santa Cruz (UCSC) Genome Browser (genome-asia.ucsc.edu/index.html) and the JASPAR database (jaspardev.genereg.net/). The NEAT1 promoter fragments containing putative STAT3-binding sites were inserted into the pGL3-basic vector (Promega Corporation). MDA-MB-231 cells were seeded in 24-well plates and cotransfected with luciferase reporter vectors, miR-124 mimics, STAT3 siRNA or negative control, and pRL-TK Renilla vector (Promega Corporation), using Lipofectamine ${ }^{\circledR} 2000$, according to the manufacturer's instructions. At $48 \mathrm{~h}$ post-transfection, the cells were lysed and the firefly and Renilla luciferase activities were detected using a Dual-Luciferase Reporter Assay System (Promega Corporation).

Cell viability assay. An MTT assay was used to measure the cell viability of MCF-7 and MDA-MB-231 lines. At $24 \mathrm{~h}$ post-transfection, cells were seeded in 96-well plates at a density of $4 \times 10^{3}$ cells/well and cultured for 24,48 and $72 \mathrm{~h}$. Then, the MTT assay was conducted as previously described (26).

Colony formation assay. At 24 h post-transfection, MCF-7 and MDA-MB-231 cells were seeded in 6-well plates at a density of 500 cells/well and cultured for 2 weeks with medium replacement every 3 days. Then the cells were fixed and stained with $0.1 \%$ crystal violet at room temperature. The colonies containing $>50$ cells were manually counted and imaged under a microscope.

Cell cycle analysis. MCF-7 and MDA-MB-231 cells were seeded in 6-well plates and transfected with oligonucleotides as indicated. At $48 \mathrm{~h}$ post-transfection, the cells were trypsinized and fixed in $70 \%$ ethanol at $4^{\circ} \mathrm{C}$ overnight. Then the cells were treated with RNase A and stained with propidium iodide (PI; Sigma-Aldrich; Merck KGaA) for $30 \mathrm{~min}$. Flow cytometry was conducted on a BD FACSCalibur flow cytometer (BD Biosciences). The results were analyzed using the ModiFit LT V3.3.11 software (Verity Software House).

Statistical analysis. Statistical analyses were performed using SPSS software (version 20.0; IBM Corp). Data are presented as the mean \pm standard deviation of at least three independent experiments. Comparisons between two groups were conducted using the Student's t-test (two-tailed). Multiple group comparisons were conducted using one-way ANOVA followed by Dunnett's or least significant difference post hoc tests. Correlation analyses between gene expression were performed with Pearson's correlation test. $\mathrm{P}<0.05$ was considered to indicate a statistically significant difference. 

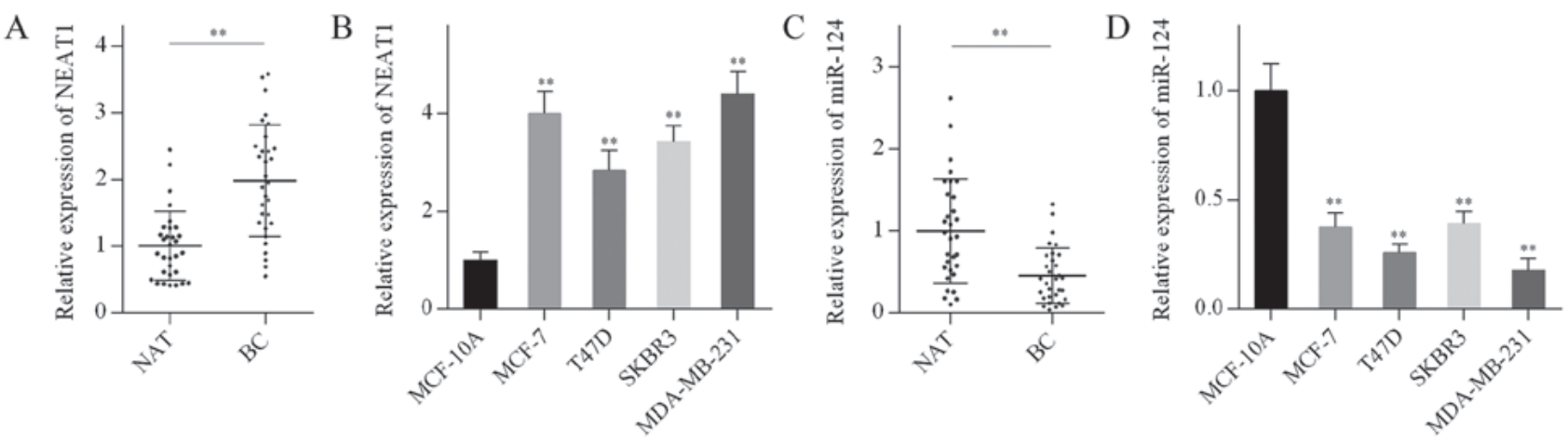

Figure 1. NEAT1 expression is increased while miR-124 expression is decreased in BC. (A) NEAT1 expression levels in BC tissues and normal breast tissues were detected by RT-qPCR. ${ }^{* *} \mathrm{P}<0.01$ (B) NEAT1 expression levels in BC cell lines and the normal breast epithelial cell line MCF-10A. Data are presented as the mean \pm SD of three independent experiments. ${ }^{* *} \mathrm{P}<0.01$ vs. MCF-10A (one-way ANOVA followed by Dunnett's test). (C) miR-124 levels in BC tissues and normal breast tissues were detected by RT-qPCR. ${ }^{* *} \mathrm{P}<0.01$ (D) miR-124 levels in BC cell lines and the normal breast epithelial cell line MCF-10A. Data are presented as the mean $\pm \mathrm{SD}$ of three independent experiments. ${ }^{* *} \mathrm{P}<0.01 \mathrm{vs}$. MCF-10A (one-way ANOVA followed by Dunnett's test). NEAT, nuclear enriched abundant transcript 1; miR-124, microRNA-124; BC, breast cancer; RT-qPCR, reverse transcription-quantitative PCR; NAT, normal adjacent breast tissues.

\section{Results}

Expression of NEAT1 and miR-124 in breast cancer. The expression levels of NEAT1 and miR-124 in breast cancer samples and cell lines were examined by RT-qPCR. NEAT1 levels in breast cancer tissues and cell lines were significantly elevated compared with normal breast tissues and MCF-10A cells (Fig. 1A and B). By contrast, miR-124 levels in breast cancer tissues and cell lines were significantly reduced compared with normal breast tissues and MCF-10A cells (Fig. 1C and D). These results suggested that NEAT1 overexpression and miR-124 downregulation may be associated with breast carcinogenesis.

NEAT1 promotes cell proliferation and cell cycle progression in breast cancer cells. To explore the biological function of NEAT1 in breast cancer cells, MCF-7 and MDA-MB-231 cells were transfected with the pcDNA-NEAT1 plasmid or NEAT1 siRNA, respectively. The transfection efficiency was determined by RT-qPCR analysis (Fig. 2A). MTT and colony formation assays demonstrated that the proliferation of MCF-7 cells was promoted by NEAT1 overexpression, whereas proliferation of MDA-MB-231 cells was inhibited by NEAT1 silencing (Fig. 2B and C). In addition, NEAT1 overexpression accelerated MCF-7 cell cycle progression, whereas G0/G1 cell cycle arrest was observed in the NEAT1-silenced MDA-MB-231 cells (Fig. 2D). These results suggested that NEAT1 promoted cell proliferation and cell cycle progression in breast cancer cells.

NEAT1 acts as a sponge of miR-124. Bioinformatics analysis based on the online database tools StarBase and miRcode indicated that NEAT1 contains a putative binding site for miR-124. The complementary binding region between miR-124 and NEAT1 is shown in Fig. 3A. To validate whether NEAT1 is a direct target of miR-124, a luciferase activity assay was performed in MDA-MB-231 cells. Luciferase activity of wild-type NEAT1 constructs (NEAT1-wt) was significantly reduced when cotransfected with miR-124 mimics. By contrast, the luciferase activity of the mutated NEAT1 construct (NEAT1-mut) was not affected by miR-124 mimics transfection, confirming the functionality of the miR-124 binding site (Fig. 3B). Furthermore, miR-124 levels were increased following NEAT1 knockdown in MCF-7 and MDA-MB-231 cells (Fig. 3C). In addition, there was a negative correlation between NEAT1 and miR-124 levels in breast cancer tissues (Fig. 3D). These results suggested that NEAT1 may function as a miR-124 sponge in breast cancer cells.

STAT3 is a direct target of $m i R-124$. To investigate the role of miR-124 in breast cancer, MCF-7 and MDA-MB-231 cells were transfected with miR-124 mimics. RT-qPCR analysis revealed that miR-124 mimics significantly elevated miR-124 levels (Fig. 4A). Additionally, miR-124 overexpression suppressed STAT3 expression in both the mRNA and protein levels (Fig. 4B and C). Bioinformatics analysis using Targetscan identified a putative miR-124 binding site in the 3'-UTR of the STAT3 mRNA (Fig. 4D). To investigate whether miR-124 directly targeted STAT3, luciferase reporter vectors containing a wild-type 3'-UTR fragment of STAT3 (STAT3-wt) or a mutant 3'-UTR fragment of STAT3 (STAT3-mut) were generated. The luciferase activity assay indicated that miR-124 overexpression dramatically reduced the luciferase activity of the STAT3-wt vector, but not the STAT3-mut vector (Fig. 4E). In addition, RT-qPCR analysis revealed that STAT3 mRNA expression levels were significantly elevated in breast cancer tissues compared with normal breast tissues (Fig. 4F), and negatively correlated with miR-124 levels in breast cancer tissues (Fig. 4G). These results suggested that STAT3 was a direct target of miR-124 in breast cancer.

miR-124 inhibits cell proliferation and induces cell cycle arrest in breast cancer cells. First, the efficiency of STAT3 overexpression was confirmed by western blot analysis; STAT3 protein expression levels were significantly elevated in cells transfected with the pcDNA-STAT3 plasmid compared with the empty vector (Fig. 5A). MTT and cell cycle analysis revealed that miR-124 overexpression in breast cancer cells decreased the cell proliferation rate and resulted in a G0/G1 phase cell cycle arrest, while these inhibitory effects were abolished by STAT3 overexpression in miR-124-overexpressing breast cancer cells (Fig. 5B and C). The results 


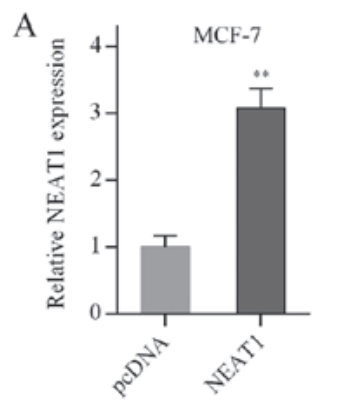

C
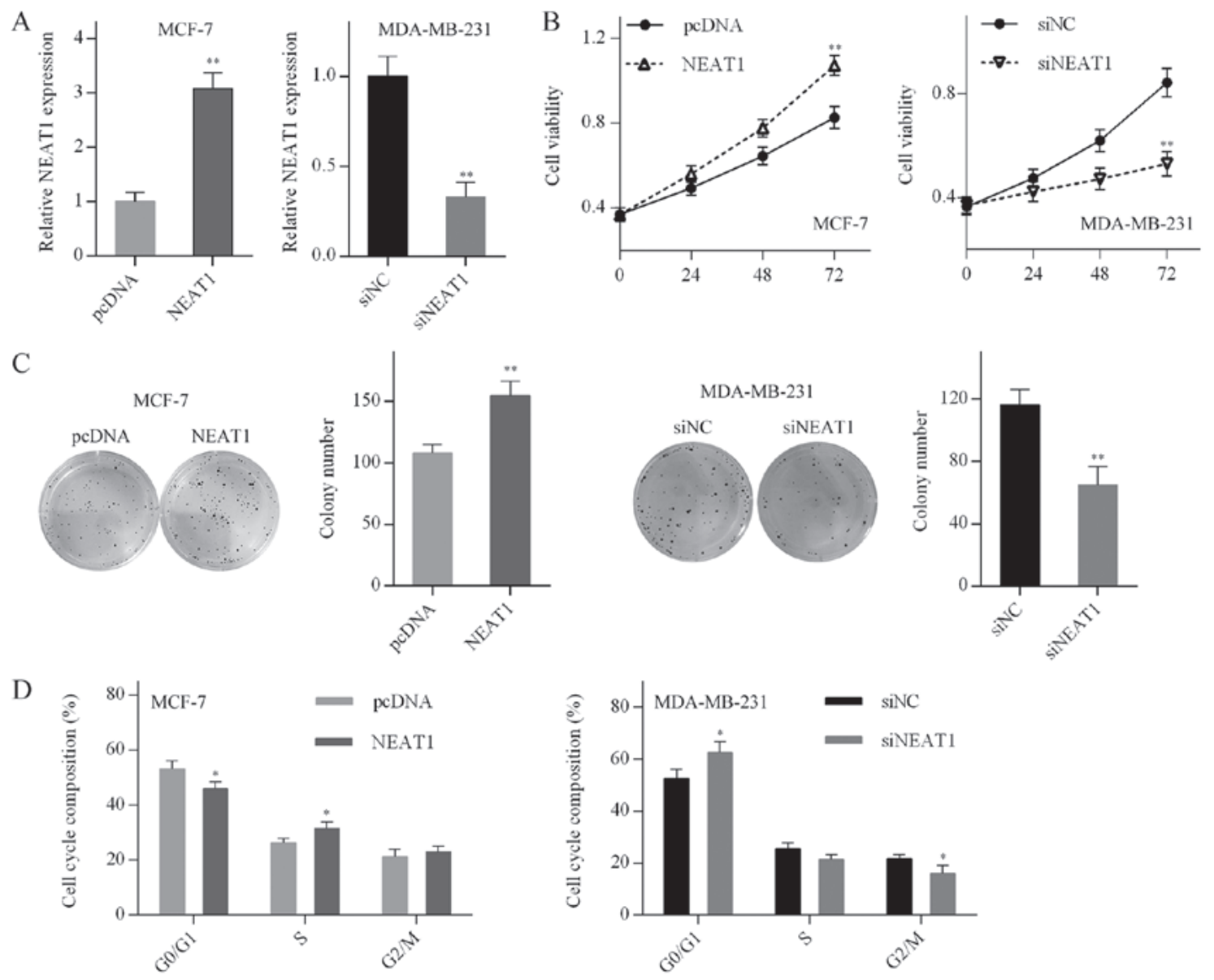

Figure 2. NEAT1 promotes cell proliferation and cell cycle progression in breast cancer cells. MCF-7 cells were transfected with the pcDNA3.1 empty vector or pcDNA-NEAT1 overexpressing plasmid. MDA-MB-231 cells were transfected with siNC and siNEAT1. (A) NEAT1 levels in MCF-7 and MDA-MB-231 cells were detected by RT-qPCR. (B) Cell viability of MCF-7 and MDA-MB-231 cells was detected by MTT assay. (C) NEAT1 overexpression promoted colony formation ability of MCF-7 cells, and NEAT1 silencing inhibited colony formation ability of MDA-MB-231 cells. (D) Cell cycle distribution of breast cancer cells was analyzed using propidium iodide staining. Data are presented as the mean $\pm \mathrm{SD}$ of at least independent triplicate experiments. "P<0.05 and ${ }^{* *} \mathrm{P}<0.01$. NEAT, nuclear enriched abundant transcript 1; si, small interfering RNA; NC, negative control; RT-qPCR, reverse transcription-quantitative PCR.
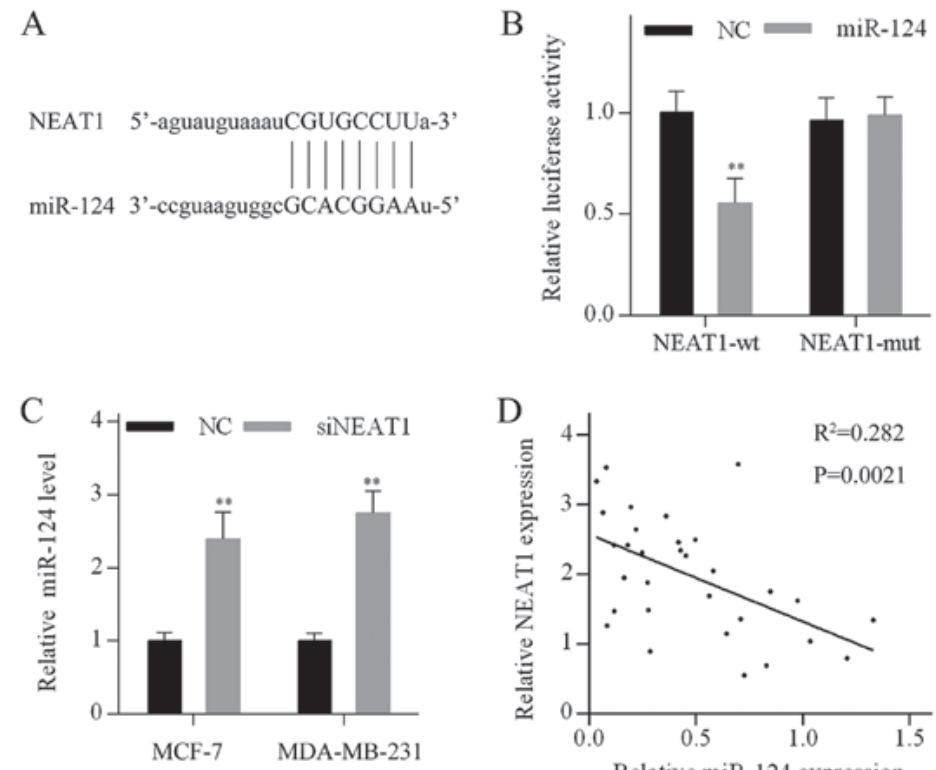

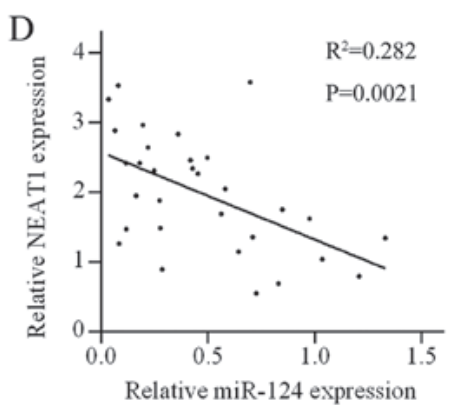

Figure 3. NEAT1 acts as a sponge of miR-124. (A) A putative miR-124-binding site on the 3 ' untranslated region of the NEAT1 transcript is shown. (B) MDA-MB-231 cells were cotransfected with the NEAT1-wt vector or NEAT1-mut vector, miR-124 mimics or negative control and the pRL-TK vector. Firefly luciferase activities were measured and normalized to Renilla luciferase activities. (C) miR-124 levels were elevated in breast cancer cells following NEAT1 silencing. (D) Relative NEAT1 levels were negatively correlated with miR-124 levels in breast cancer tissues. Data are presented as the mean \pm SD of at least independent triplicate experiments. ${ }^{* *} \mathrm{P}<0.01$. NEAT, nuclear enriched abundant transcript 1; miR-124, microRNA-124; wt, wild-type; mut, mutant; NC, negative control; si, small interfering. 


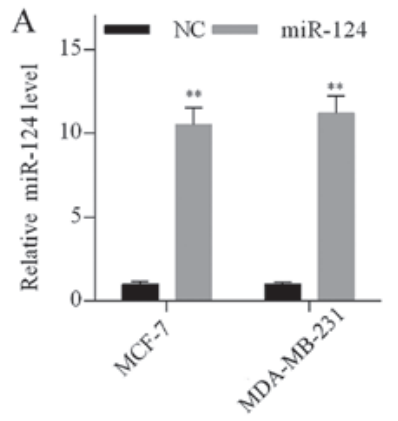

$\mathrm{D}$

STAT3 5'-ccugeccuguggGUGCCUUa-3'

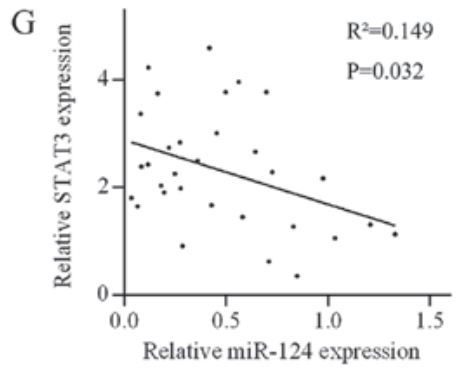

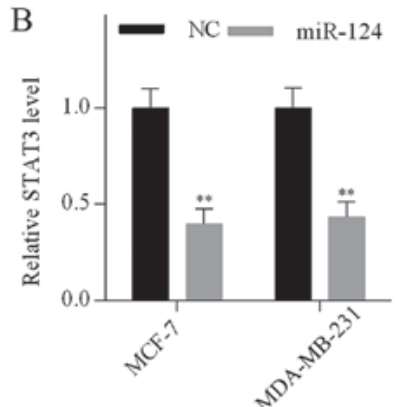

$\mathrm{C}$
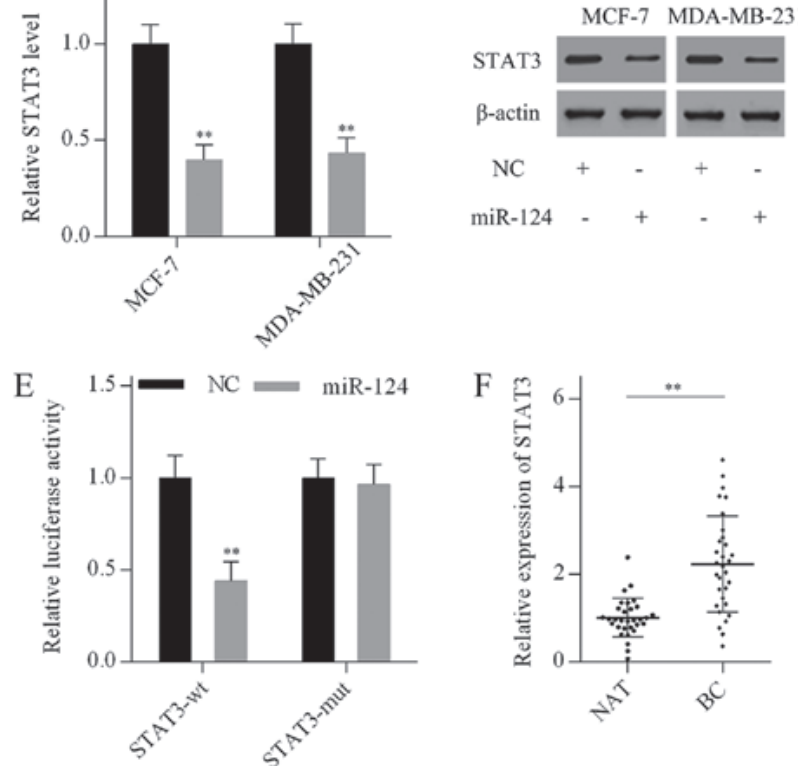

Figure 4. STAT3 is a direct target of miR-124. (A) miR-124 levels in breast cancer cells were analyzed by RT-qPCR following miR-124 mimics transfection. (B) STAT3 mRNA expression levels were detected by RT-qPCR following miR-124 overexpression. (C) STAT3 protein expression levels were analyzed by western blotting following miR-124 overexpression. (D) The predicted miR-124 binding site in the 3'-untranslated region of the STAT3 mRNA is shown. (E) MDA-MB-231 cells were cotransfected with the STAT3-wt vector or STAT3-mut vector, miR-124 mimics or negative control and pRL-TK vector. Firefly luciferase activities were normalized to Renilla luciferase activities. (F) STAT3 levels in breast cancer and normal breast tissues were detected by RT-qPCR. (G) Relative STAT3 levels were negatively correlated with miR-124 levels in breast cancer tissues. Data are shown as the mean \pm SD of three independent experiments. ${ }^{* *} \mathrm{P}<0.01$. miR-124, microRNA-124; RT-qPCR, reverse transcription-quantitative PCR; wt, wild-type; mut, mutant; NC, negative control; NAT, normal adjacent breast tissues; BC, breast cancer tissues.
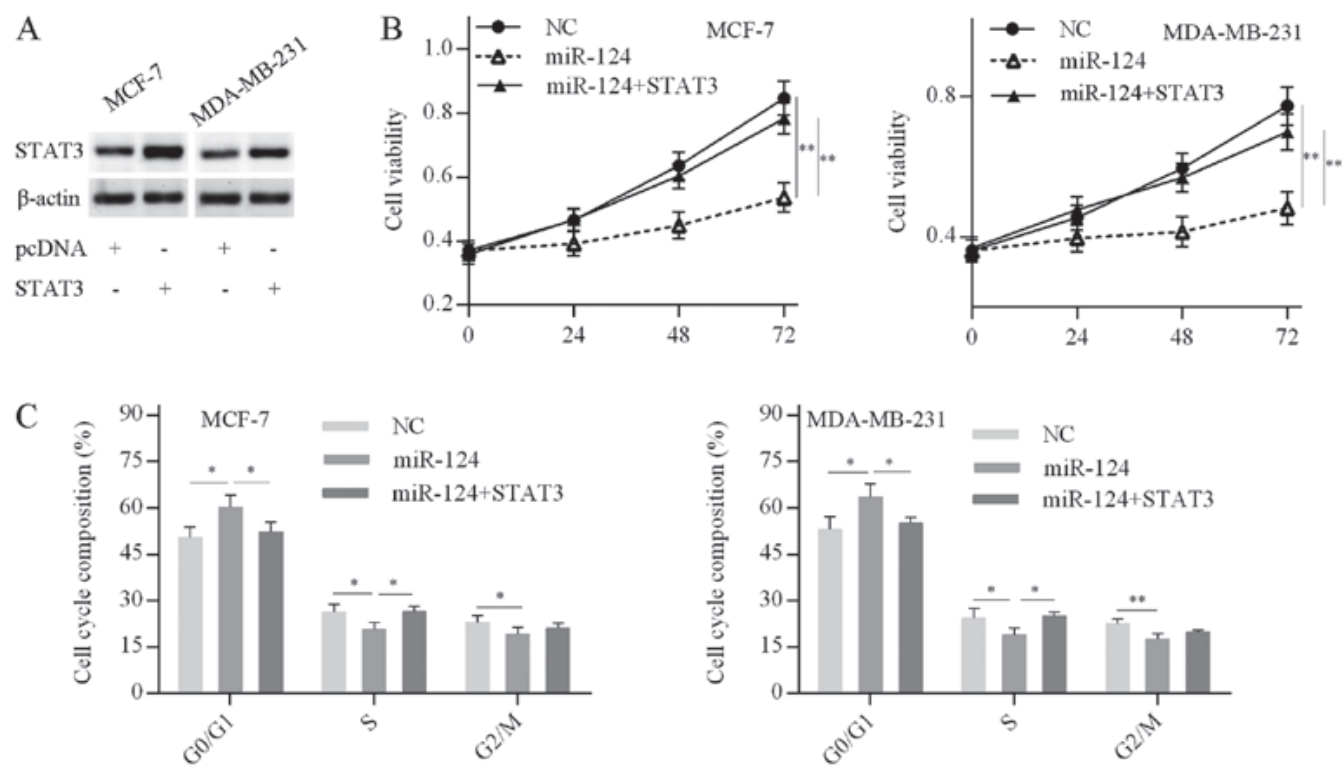

Figure 5. miR-124 inhibits breast cancer cell proliferation and cell cycle progression via targeting STAT3. MCF-7 and MDA-MB-231 cells were transfected with miR-124 mimics, pcDNA-STAT3 plasmid and corresponding negative control as indicated. (A) STAT3 overexpression in breast cancer cells was confirmed by western blotting. (B) Cell viability of breast cancer cells was detected by MTT assay. (C) Cell cycle distribution of breast cancer cells was analyzed using propidium iodide staining. Data are shown as the mean $\pm \mathrm{SD}$ of three independent experiments. " $\mathrm{P}<0.05$ and ${ }^{* * *} \mathrm{P}<0.01$, with comparisons indicated by lines (one-way ANOVA followed by least significant difference test). miR-124, microRNA-124; NC, negative control. 

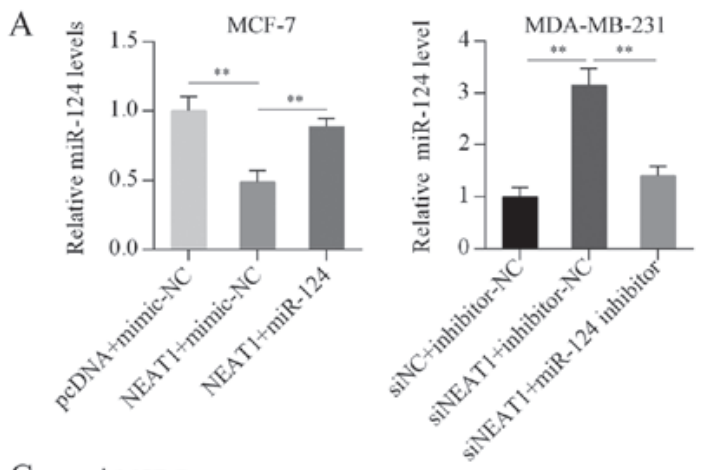

B
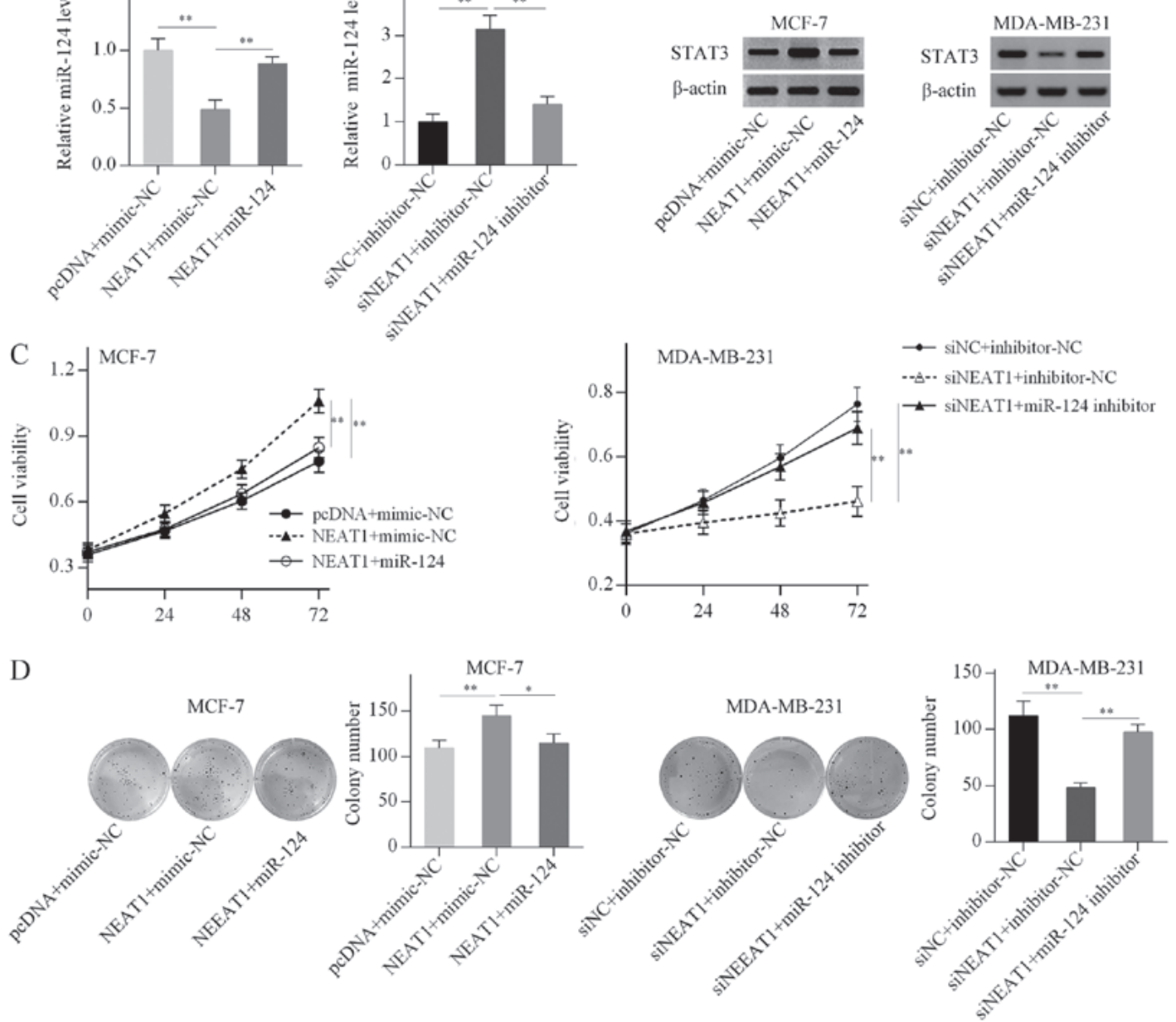

$\mathrm{E}$
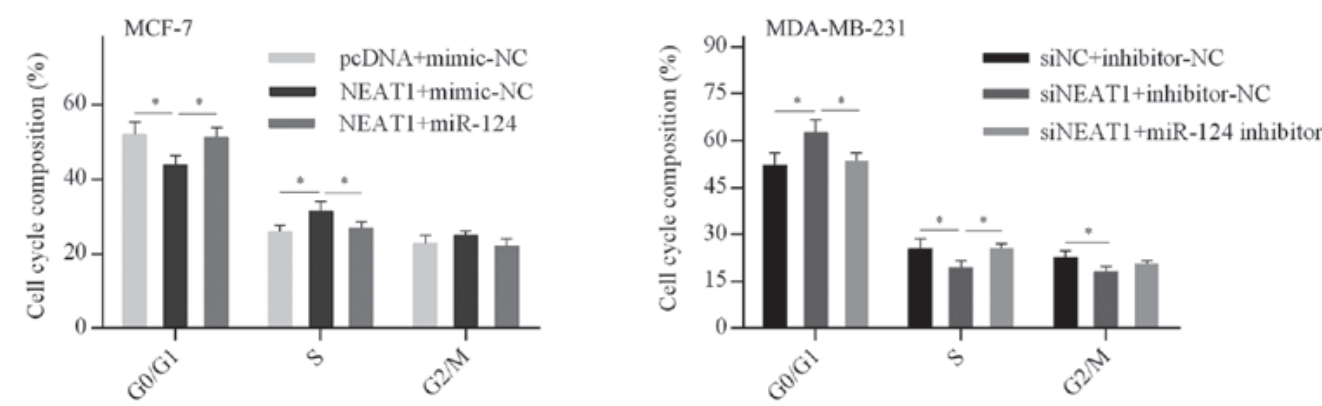

Figure 6. NEAT1 regulates cell proliferation and cell cycle of breast cancer cells via targeting the miR-124/STAT3 axis. MCF-7 cells were transfected with pcDNA-NEAT1, miR-124 mimics and corresponding NC, and MDA-MB-231 cells were transfected with siNEAT1, miR-124 inhibitors or corresponding NC, as indicated. (A) miR-124 levels in breast cancer cells were detected by reverse transcription-quantitative PCR. (B) STAT3 protein levels in breast cancer cells were detected by western blotting. (C) Cell viability of breast cancer cells was detected by MTT assay. (D) Colony formation ability of breast cancer cells was analyzed by counting colony number under a microscope. (E) Cell cycle distribution of breast cancer cells was analyzed using propidium iodide staining. Data are shown as the mean $\pm \mathrm{SD}$ of three independent experiments. ${ }^{*} \mathrm{P}<0.05$ and ${ }^{* *} \mathrm{P}<0.01$, with comparisons indicated by lines (one-way ANOVA followed by least significant difference test). NEAT, nuclear enriched abundant transcript 1; miR-124, microRNA-124; NC, negative control; si, small interfering RNA.

suggested that miR-124 inhibits breast cancer cell proliferation and cell cycle progression by targeting STAT3.

NEAT1 promotes breast cancer cell growth via targeting the miR-124/STAT3 axis. Next, the present study investigated whether NEAT1 acts as a ceRNA to increase STAT3 expression in breast cancer cells. RT-qPCR analysis demonstrated that miR-124 overexpression reversed the NEAT1-mediated inhibitory effect on miR-124 expression in MCF-7 cells, and miR-124 depletion weakened the NEAT1 silencing-induced
miR-124 upregulation in MDA-MB-231 cells (Fig. 6A). Western blot analysis further revealed that NEAT1 overexpression increased STAT3 protein expression in MCF-7 cells, while this effect was weakened by miR-124 overexpression (Fig. 6B). By contrast, STAT3 expression was decreased in NEAT1-silenced MDA-MB-231 cells, and miR-124 knockdown alleviated the inhibitory effect of NEAT1 silencing on STAT3 expression (Fig. 6B). These results suggested that NEAT1 positively regulated STAT3 expression by acting as a ceRNA that sponges miR-124 in breast cancer cell lines. 
A

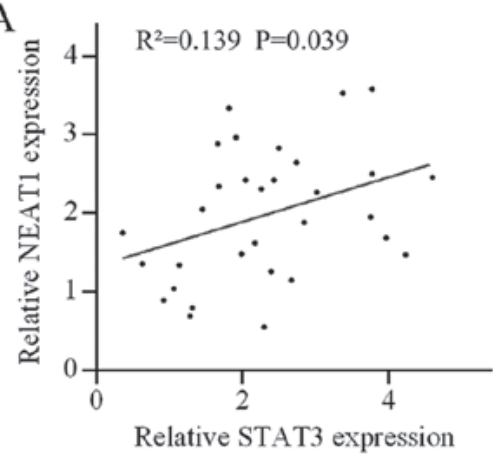

D

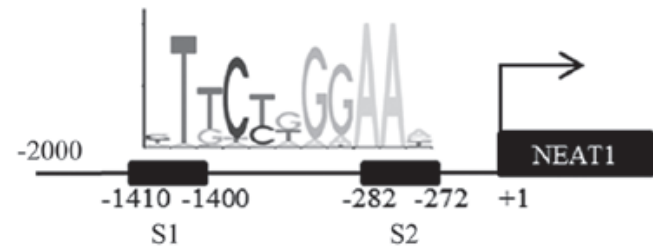

B

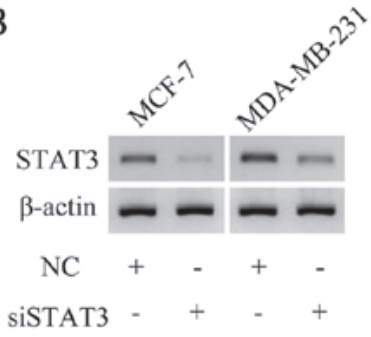

C

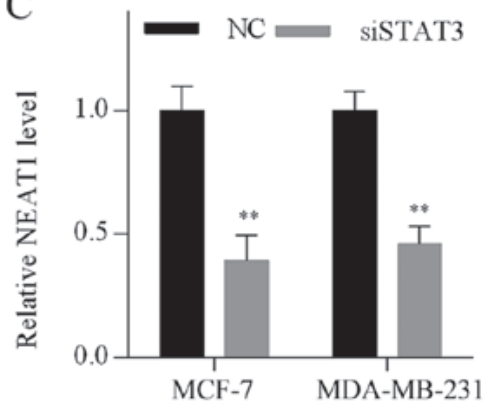

E

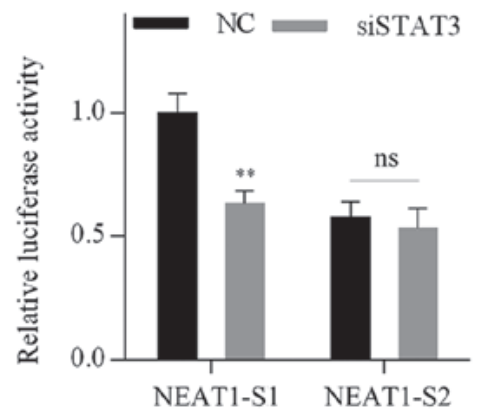

Figure 7. NEAT1 is inhibited by STAT3 silencing. (A) Relative STAT3 mRNA expression levels were positively correlated with NEAT1 expression levels in breast cancer tissues. (B) STAT3 siRNA silencing in breast cancer cells was confirmed by western blotting. (C) NEAT1 levels in breast cancer cells were detected by reverse transcription-quantitative PCR following STAT3 silencing. (D) Schematic graph of the binding sites (score $>10$ ) for STAT3 in the NEAT1 promoter. (E) Luciferase activities of the two fragments containing the S1 or S2 binding sites following STAT3 silencing. Data are shown as the mean \pm SD of three independent experiments. * P<0.01. NEAT, nuclear enriched abundant transcript 1; si, small interfering RNA; NC, negative control; ns, not significant.

Subsequently, the present study investigated whether NEAT1 promoted the proliferation and cell cycle progression of breast cancer cells by targeting the miR-124/STAT3 axis. Functional analysis revealed that miR-124 overexpression effectively reduced NEAT1-induced cell proliferation and cell cycle progression in MCF-7 cells (Fig. 6C-E). Conversely, the inhibitory effects of NEAT1 silencing on cell proliferation and cell cycle were abrogated by miR-124 depletion in MDA-MB-231 cells (Fig. 6C-E). These results suggested that NEAT1 promoted breast cancer cell growth by targeting the miR-124/STAT3 axis.

NEAT1 is inhibited by STAT3 silencing. To explore the crosstalk between NEAT1 and STAT3, their correlation in breast cancer specimens was evaluated. NEAT1 levels were positively correlated with STAT3 mRNA expression levels (Fig. 7A). Then, STAT3 expression was silenced in MCF-7 and MDA-MB-231 cells using specific siRNA (Fig. 7B). RT-qPCR analysis demonstrated that NEAT1 expression levels were decreased following STAT3 silencing (Fig. 7C). Furthermore, multiple putative STAT3 binding sites were identified in the NEAT1 promoter by bioinformatics analysis using the UCSC (genome-asia.ucsc.edu/index.html) and JASPAR (jaspardev. genereg.net/) web-based tools. Two binding sites (S1 and S2) with prediction scores $>10$ are shown in Fig. 7D. Subsequently, two fragments containing S1 or S2 were cloned into luciferase reporter vectors. The vectors were cotransfected into MDA-MB-231 cells with STAT3 siRNA or negative control siRNA. Luciferase activity analysis revealed that STAT3 silencing significantly reduced the luciferase activity of fragment $\mathrm{S} 1$, whereas the luciferase activity of fragment S2 was not affected (Fig. 7E). These results demonstrated that STAT3 positively regulated NEAT1 transcription, and thus formed a positive feedback loop in breast cancer cells.

\section{Discussion}

Emerging evidence has revealed that lncRNAs have a pivotal role in the regulation of physiological and pathological processes, including in breast cancer (6). According to bioinformatics analysis, in the present study, miR-124 was predicted to directly target both NEAT1 and STAT3. The association between NEAT1 and miR-124 in breast cancer has not been elucidated in previous studies. As a result, the present study focused on miR-124 as the target gene of NEAT1. The results demonstrated that NEAT1 and STAT3 expression levels were elevated in breast cancer, whereas miR-124 was significantly reduced. Thus, it was speculated that NEAT1 may be involved in breast cancer progression by modulating the miR-124/STAT3 axis.

Accumulating evidence has suggested that NEAT1 has a critical role in carcinogenesis (10). For example, NEAT1 is highly expressed in hepatocellular carcinoma (HCC) and promotes the proliferation of HCC cells by regulating the miR-129/valosin containing protein/inhibitor of $\kappa \mathrm{B}$ kinase axis (27). NEAT1 has been identified to be an indicator of diagnosis and prognosis in colorectal cancer (28). Furthermore, high NEAT1 expression was reported to be associated with TNM stage and overall survival in breast cancer $(29,30)$. In the present study, it was demonstrated that NEAT1 expression was elevated in breast cancer tissues and cell lines, as previously described $(10,29,30)$. In addition, NEAT1 overexpression 
promoted cell proliferation and cell cycle progression in breast cancer cells, whereas NEAT1 silencing led to a reduced proliferation rate and cell cycle arrest at G0/G1 phase.

NEAT1 has been reported to regulate gene expression by a variety of mechanisms (10). NEAT1 forms a complex with forkhead box protein N3 and paired amphipathic helix protein SIN3A, and thus represses transacting T-cell-specific transcription factor GATA3 expression, which is involved in epithelial-mesenchymal transition (31). Additionally, NEAT1 epigenetically represses E-cadherin expression through interaction with histone-lysine N-methyltransferase EHMT2/ DNA (cytosine-5)-methyltransferase 1/Snail complex (32). Previous studies have identified a new regulatory mechanism in which lncRNAs act as endogenous sponges of miRNAs $(33,34)$. NEAT1 acts as a ceRNA to positively regulate histone-lysine $\mathrm{N}$-methyltransferase EZH2 expression by sponging miR-101 in breast cancer cells (35). In the current study, miR-124 expression was shown to be negatively correlated with NEAT1 and STAT3 expression in breast cancer tissues, suggesting potential crosstalk between miR-124 and the other two RNAs. Furthermore, bioinformatics prediction analysis indicated that NEAT1 and STAT3 might be potential direct targets of miR-124. The luciferase activity assay and RT-qPCR analysis demonstrated that miR-124 directly targeted NEAT1 and STAT3 in breast cancer cells. However, no significant correlation between NEAT1, miR-124 and STAT3 expression in breast cancer cell lines was identified (data not shown). This may be attributed to the limited number of breast cancer cell lines used in the present study.

In HCC, NEAT1 acts as a ceRNA to increase STAT3 expression by sponging miR-485, resulting in enhanced cancer progression (36). Additionally, NEAT1 promotes gastric cancer development by targeting miR-506/STAT3 axis (37). In the present study, it was confirmed that STAT3 protein levels were elevated following NEAT1 overexpression, and partially attenuated by miR-124overexpression in NEAT1-overexpressing breast cancer cells. The results suggested that NEAT1 acted as a ceRNA to increase STAT3 expression by sponging miR-124 in breast cancer. Subsequently, the findings of the present study demonstrated that the effects of NEAT1 on the proliferation and cell cycle of breast cancer cells were attenuated by miR-124 overexpression. Moreover, miR-124 overexpression inhibited the growth of breast cancer cells by targeting STAT3. Taken together, these findings indicated that NEAT1 may promote the growth of breast cancer cells by targeting the miR-124/STAT3 axis.

Previous studies have demonstrated that STAT3 is constitutively activated in various types of cancer, including breast cancer (38). Phosphorylated STAT3 is translocated into the nucleus and binds to the consensus promoter sequence of target genes to initiate transcription (39). Recent studies have demonstrated that IncRNAs are regulated by the interleukin-6 (IL6)/STAT3 pathway in cancers. For example, a set of IncRNAs were induced by IL-6-activated STAT3 in multiple myeloma cells (40). In glioma cells, activation of the EGF receptor pathway positively regulated NEAT1 expression via STAT3 and NF- $\mathrm{KB}$ (p65) (41). In addition, IL-6 promoted NEAT1 transcription via STAT3 and histone 3 lysine 4 trimethylation in HCC cells (42). The current study identified a positive correlation between NEAT1 and STAT3 expression levels in breast cancer tissues. Furthermore, it was demonstrated that STAT3 silencing reduced NEAT1 expression levels by inhibiting the promoter activity in breast cancer cells. Therefore, NEAT1 and STAT3 formed a positive feedback loop mediated by miR-124.

In conclusion, the findings of the present study demonstrated that NEAT1 positively regulated STAT3 expression by sponging miR-124 in breast cancer cells. Furthermore, a positive feedback loop between NEAT1 and STAT3 that contributed to breast cancer cell growth was identified. These results increase the understanding of the molecular mechanisms underlying breast cancer development and indicate the possibility of developing NEAT1 as a potential target in breast cancer treatment.

\section{Acknowledgements}

Not applicable.

\section{Funding}

No funding was received.

\section{Availability of data and materials}

All data and materials involved in this study are available from the corresponding author on reasonable request.

\section{Authors' contributions}

GY, YP and JL were responsible for the study conception and design. JW, XL, CW and MW performed the majority of the experiments. YP and JL drafted the manuscript. All the authors read and approved the final manuscript.

\section{Ethics approval and consent to participate}

This study was approved by the Ethics Committee of Xi'an Jiaotong University First Affiliated Hospital and each patient provided written informed consent.

\section{Patient consent for publication}

Not applicable.

\section{Competing interests}

The authors declare that they have no competing interests.

\section{References}

1. Siegel RL, Miller KD 1. Siegel RL, Miller KD and Jemal A: Cancer statistics, 2016. CA Cancer J Clin 66: 7-30, 2016.

2. Cancer Genome Atlas N; Cancer Genome Atlas Network: Comprehensive molecular portraits of human breast tumours. Nature 490: 61-70, 2012.

3. Torre LA, Siegel RL, Ward EM and Jemal A: Global cancer incidence and mortality rates and trends - an update. Cancer Epidemiol Biomarkers Prev 25: 16-27, 2016.

4. Huarte M: The emerging role of lncRNAs in cancer. Nat Med 21: 1253-1261, 2015

5. Fatica A and Bozzoni I: Long non-coding RNAs: New players in cell differentiation and development. Nat Rev Genet 15: 7-21, 2014.

6. Yang G, Lu X and Yuan L: LncRNA: A link between RNA and cancer. Biochim Biophys Acta 1839: 1097-1109, 2014. 
7. Zheng R, Lin S, Guan L, Yuan H, Liu K, Liu C, Ye W, Liao Y, Jia J and Zhang R: Long non-coding RNA XIST inhibited breast cancer cell growth, migration, and invasion via miR-155/CDX1 axis. Biochem Biophys Res Commun 498: 1002-1008, 2018.

8. Xue X, Yang YA, Zhang A, Fong KW, Kim J, Song B, Li S, Zhao JC and Yu J: LncRNA HOTAIR enhances ER signaling and confers tamoxifen resistance in breast cancer. Oncogene 35: 2746-2755, 2016

9. Peng F, Li TT, Wang KL, Xiao GQ, Wang JH, Zhao HD, Kang ZJ, Fan WJ, Zhu LL, Li M, et al: H19/let-7/LIN28 reciprocal negative regulatory circuit promotes breast cancer stem cell maintenance. Cell Death Dis 8: e2569, 2017.

10. Yu X, Li Z, Zheng H, Chan MT and Wu WK: NEAT1: A novel cancer-related long non-coding RNA. Cell Prolif 50: 50, 2017.

11. Jiang X, Zhou Y, Sun AJ and Xue JL: NEAT1 contributes to breast cancer progression through modulating miR-448 and ZEB1. J Cell Physiol 233: 8558-8566, 2018.

12. Bartel DP: MicroRNAs: Genomics, biogenesis, mechanism, and function. Cell 116: 281-297, 2004.

13. Di Leva G, Garofalo M and Croce CM: MicroRNAs in cancer Annu Rev Pathol 9: 287-314, 2014.

14. Pang Y, Liu J, Li X, Xiao G, Wang H, Yang G, Li Y, Tang SC Qin S, Du N, et al: MYC and DNMT3A-mediated DNA methylation represses microRNA-200b in triple negative breast cancer. J Cell Mol Med 22: 6262-6274, 2018.

15. Jiang L, Lin T, Xu C, Hu S, Pan Y and Jin R: miR-124 interacts with the Notch1 signalling pathway and has therapeutic potential against gastric cancer. J Cell Mol Med 20: 313-322, 2016.

16. Lu Y, Yue X, Cui Y, Zhang J and Wang K: MicroRNA-124 suppresses growth of human hepatocellular carcinoma by targeting STAT3. Biochem Biophys Res Commun 441: 873-879, 2013.

17. Wu DH, Liang H, Lu SN, Wang H. Su Z-L. Zhang L, Ma J-Q, Guo M, Tai S and Yu S: miR-124 suppresses pancreatic ductal adenocarcinoma growth by regulating monocarboxylate transporter 1-mediated cancer lactate metabolism. Cell Physiol Biochem 50: 924-935, 2018.

18. Wang M, Meng B, Liu Y, Yu J, Chen Q and Liu Y: miR-124 inhibits growth and enhances radiation-induced apoptosis in non-small cell lung cancer by inhibiting STAT3. Cell Physiol Biochem 44: 2017-2028, 2017.

19. Liang YJ, Wang QY, Zhou CX, Yin QQ, He M, Yu XT, Cao DX, Chen GQ, He JR and Zhao Q: miR-124 targets Slug to regulate epithelial-mesenchymal transition and metastasis of breast cancer. Carcinogenesis 34: 713-722, 2013.

20. Cai WL, Huang WD, Li B, Chen TR, Li ZX, Zhao CL, Li HY, Wu YM, Yan WJ and Xiao JR: microRNA-124 inhibits bone metastasis of breast cancer by repressing Interleukin-11. Mol Cancer 17: 9, 2018

21. Chen SM, Chou WC, Hu LY, Hsiung CN, Chu HW, Huang YL, Hsu HM, Yu JC and Shen CY: The effect of microRNA-124 overexpression on anti-tumor drug sensitivity. PLoS One 10: e0128472, 2015.

22. Fu Y and Xiong J: MicroRNA-124 enhances response to radiotherapy in human epidermal growth factor receptor 2-positive breast cancer cells by targeting signal transducer and activator of transcription 3. Croat Med J 57: 457-464, 2016.

23. Holliday DL and Speirs V: Choosing the right cell line for breast cancer research. Breast Cancer Res 13: 215, 2011.

24. Pang Y, Liu J, Li X, Zhang Y, Zhang B, Zhang J, Du N, Xu C, Liang R, Ren $\mathrm{H}$, et al: Nano Let-7b sensitization of eliminating esophageal cancer stem-like cells is dependent on blockade of Wnt activation of symmetric division. Int J Oncol 51: 1077-1088, 2017.

25. Livak KJ and Schmittgen TD: Analysis of relative gene expression data using real-time quantitative PCR and the 2(-Delta Delta C(T)) Method. Methods 25: 402-408, 2001.
26. Liu J, Li X, Wang M, Xiao G, Yang G, Wang H, Li Y, Sun X, Qin S, Du N, et al: A miR-26a/E2F7 feedback loop contributes to tamoxifen resistance in ER-positive breast cancer. Int J Oncol 53: $1601-1612,2018$

27. Fang L, Sun J, Pan Z, Song Y, Zhong L, Zhang Y, Liu Y, Zheng X and Huang P: Long non-coding RNA NEAT1 promotes hepatocellular carcinoma cell proliferation through the regulation of miR-129-5p-VCP-IкB. Am J Physiol Gastrointest Liver Physiol 313: G150-G156, 2017.

28. Li Y, Li Y, Chen W, He F, Tan Z, Zheng J, Wang W, Zhao Q and Li J: NEAT expression is associated with tumor recurrence and unfavorable prognosis in colorectal cancer. Oncotarget 6: 27641-27650, 2015

29. Zhao D, Zhang Y, Wang N and Yu N: NEAT1 negatively regulates miR-218 expression and promotes breast cancer progression. Cancer Biomark 20: 247-254, 2017.

30. Li X, Wang S, Li Z, Long X, Guo Z, Zhang G, Zu J, Chen Y and Wen L: The lncRNA NEAT1 facilitates cell growth and invasion via the miR-211/HMGA2 axis in breast cancer. Int J Biol Macromol 105: 346-353, 2017.

31. Li W, Zhang Z, Liu X, Cheng X, Zhang Y, Han X, Zhang Y, Liu S, Yang J, Xu B, et al: The FOXN3-NEAT1-SIN3A repressor complex promotes progression of hormonally responsive breast cancer. J Clin Invest 127: 3421-3440, 2017.

32. Li Y and Cheng C: Long noncoding RNA NEAT1 promotes the metastasis of osteosarcoma via interaction with the G9a-DNMT1-Snail complex. Am J Cancer Res 8: 81-90, 2018.

33. Salmena L, Poliseno L, Tay Y, Kats L and Pandolfi PP: A ceRNA hypothesis: The Rosetta Stone of a hidden RNA language? Cell 146: 353-358, 2011

34. Bayoumi AS, Sayed A, Broskova Z, Teoh JP, Wilson J, Su H, Tang YL and Kim IM: Crosstalk between long noncoding RNAs and microRNAs in health and disease. Int J Mol Sci 17: 356, 2016

35. Qian K, Liu G, Tang Z, Hu Y, Fang Y, Chen Z and Xu X: The long non-coding RNA NEAT1 interacted with miR-101 modulates breast cancer growth by targeting EZH2. Arch Biochem Biophys 615: 1-9, 2017.

36. Zhang XN, Zhou J and Lu XJ: The long noncoding RNA NEAT1 contributes to hepatocellular carcinoma development by sponging miR-485 and enhancing the expression of the STAT3. J Cell Physiol 233: 6733-6741, 2018.

37. Tan HY, Wang C, Liu G and Zhou X: Long noncoding RNA NEAT1-modulated miR-506 regulates gastric cancer development through targeting STAT3. J Cell Biochem 120: 4827-4836, 2019.

38. Banerjee $\mathrm{K}$ and Resat $\mathrm{H}$ : Constitutive activation of STAT3 in breast cancer cells: A review. Int J Cancer 138: 2570-2578, 2016.

39. Srivastava J and DiGiovanni J: Non-canonical Stat3 signaling in cancer. Mol Carcinog 5: 1889-1898, 2016.

40. Binder S, Hösler N, Riedel D, Zipfel I, Buschmann T, Kämpf C, Reiche K, Burger R, Gramatzki M, Hackermüller J, et al: STAT3-induced long noncoding RNAs in multiple myeloma cells display different properties in cancer. Sci Rep 7: 7976 , 2017.

41. Chen Q, Cai J, Wang Q, Wang Y, Liu M, Yang J, Zhpu J, Kang C, Li $\mathrm{M}$ and Jiang C: Long noncoding RNA NEAT1, regulated by the EGFR pathway, contributes to glioblastoma progression through the WNT/beta-catenin pathway by scaffolding EZH2. Clin Cancer Res 24: 684-695, 2018.

42. Wang S, Zhang Q, Wang Q, Shen Q, Chen X, Li Z, Zhou Y, Hou J, $\mathrm{Xu} \mathrm{B}, \mathrm{Li} \mathrm{N}$, et al: NEAT1 paraspeckle promotes human hepatocellular carcinoma progression by strengthening IL-6/STAT3 signaling. OncoImmunology 7: e1503913,2018. 